\title{
Interstellar Scintillation of Radio Sources as a Probe for Investigations of the Local Interstellar Medium
}

\author{
N. Bochkarev ${ }^{1}$ and M. Ryabov ${ }^{2}$
}

1 Sternberg Astronomical Institute, Universitetskij prosp. 13, Moscow, 119899, Russia

2 Odessa Observatory URAN-4 of Radioastronomical Institute of National Academy of Science, Pushkinskaya str. 37, Odessa, 270011, Ukraine

\begin{abstract}
A possibility of obtaining information on small scale inhomogeneities of the electron component of the local interstellar medium (LISM) is investigated using interstellar scintillations of extragalactic radio sources. We analyse Culgoora array observational data on variability of 190 extragalactic radio sources, covering most of the sky, at 80 and $160 \mathrm{MHz}$. The variability at time scales from 1 month to 15 years is interpreted as refractive interstellar scintillations in fast-moving nearby (less than $150 \mathrm{pc}$ ) hot gas near shock waves in the LISM. All-sky map of scintillation indices $m$ averaged over $3-5$ sources closest to one another shows several $m$ maxima. Two of the 3 most pronounced maxima are probably connected with Loop I; the third one coincides with the soft X-ray $(0.1-0.3 \mathrm{keV})$ background maximum near the South Galactic Pole. Other, less certain, $m$ maxima probably correspond to the Orion star-formation region and to a soft X-ray maximum near the North Galactic Pole. The "free-of-gas" tunnel in the direction $l=240^{\circ}$ corresponds to low values of $m$. The estimated time scale of interstellar scintillations on the above-mentioned LISM structures is in agreement with that of the observed radio-source variations.
\end{abstract}

\section{Introduction}

Interstellar scintillations of radio sources resulting from small-scale inhomogeneities of interstellar matter's refractive coefficient (Rickett 1986) can yield information about plasma turbulence near shock fronts (Pikelner and Tsytovich 1969). Here, the thin scattering screen approximation may be applied.

\section{Observational Data and Analysis}

We used Culgoora (Australia) 1970-1984 array observations of extragalactic radio sources at 80 and $160 \mathrm{MHz}$ (Slee and Siegman 1988). During 15 years, 412 sources covering homogeneously most of the sky (with the exception of a northern sky section) were observed repeatedly, in two or more 4-week-long series. 190 sources proved variable at least at one frequency and only 27 , at both frequencies. Slee and Siegman (1988) give many arguments in favor 
of interstellar origin of the variations. They published measured scintillation variability indices $m$ for the time scale of 1 month $(m 1)$ and from one to several years $(m 12)$.

The Slee \& Siegman survey provides no information about the energy emitted by scintillating point components of individual sources. The $m$ value for any source, however, depends not only on interstellar matter properties, but also on the source structure. Therefore, we have drawn an all-sky map for the interstellar scintillation indices $m$ averaged over 3-5 neighboring sources (Fig. 1). Moreover, we used the averaged $m$ values for the two time scales ( $m 1$ and $m 12$ ) and the data for both frequencies ( 80 and $160 \mathrm{MHz}$ ). Typical angular distances between sources are about $15^{\circ}$. Thus, we hope the averaging will allow us to perceive interstellar formations of angular size not less than 20-30 degrees. We checked the sensitivity of our results to the input data used varying the data combinations ( $m 1$ or/and $m 12 ; 80$ or/and $160 \mathrm{MHz}$ ) and the number of averaged sources, and also removing the data for the 3 sources with the extreme values of $m$. In all cases, the main maxima are present for the majority of the data combinations and conserve their positions within our angular resolution (about $20^{\circ}-30^{\circ}$ ), with slight variations of sizes and positions of the mapped structures .

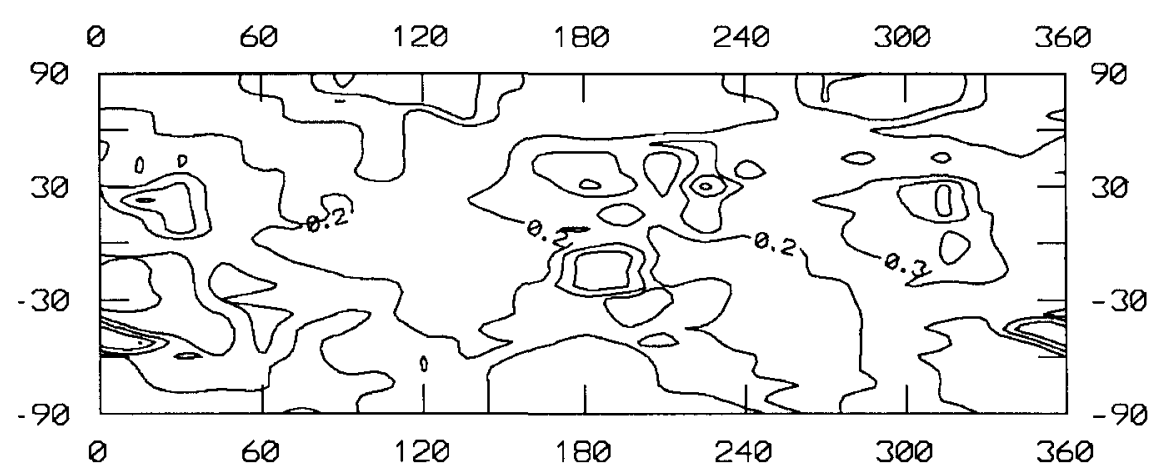

Fig. 1. The all-sky map of isolines of scintillation indices, $m$, for sources from Slee and Siegman (1988) survey, in galactic coordinates. The map is drawn using data averaged over 3 neighboring sources and over $m 1$ and $m 12$ for $80 \mathrm{MHz}$ and $160 \mathrm{MHz}$. Isolines are from $m=0.2$, with 0.1 increment.

\section{Results and Interpretation}

We found 3 clear maxima, virtually independent of the data combinations and ways of averaging, in the all-sky distribution of interstellar scintillation 
Table 1. Table 1. Galactic coordinates and possible identifications of the features in the map of interstellar scintillation index distribution

\begin{tabular}{ll}
\hline \multicolumn{1}{c}{ Features } & Identification \\
\hline $1.0^{\circ}<l<30^{\circ} ; 0^{\circ}<b<30^{\circ}$ & Clear maxima \\
2. $300^{\circ}<l<330^{\circ} ; 0^{\circ}<b<30^{\circ}$ & Loop I \\
L. $-20^{\circ}<l<20^{\circ} ;-60^{\circ}<b<-40^{\circ}$ & Soop I \\
& (B and C bands, McCammon et al. 1983) \\
\hline \multicolumn{1}{c}{ Other possible maxima } \\
\hline $4.170^{\circ}<l<220^{\circ} ; 30^{\circ}<b<45^{\circ}$ & Northern maximum of soft X-ray BG \\
$5.180^{\circ}<l<200^{\circ} ;-20^{\circ}<b<0^{\circ}$ & Orion star-formation region \\
$6 . l \approx 120^{\circ}$ and $300^{\circ} b>75^{\circ}$ & Soft X-ray BG filament near NGP \\
& (B and C bands, McCammon et al. 1983) \\
\hline $7 . l \approx 240^{\circ}$ low southern b & Minimum of $m$ \\
\hline
\end{tabular}

indices (Table 1). Three other maxima, though probably real, are not as definite as the three first ones. In all the cases, a minimum of $m$ is present near the galactic longitude $l=240^{\circ}$ in the Southern hemisphere, but the data for this direction is too scarce to allow definite conclusions.

We assume that the revealed maxima of $m$ can be identified with maxima of the soft X-ray background radiation distribution (B, C, and $\mathrm{M}$ bands in the survey by McCammon et al. 1983). The maxima 1 and 2 from Table 1 are overlapping a considerable part of the two brightest X-ray filaments in the M1 band (440-930 eV) map corresponding to Loop I, the old SNR expanding inside the coronal gas in a cavern formed around the Sco-Cen stellar association (Bochkarev 1987b, 1990,).

Another marked $m$ maximum (number 3 ) obviously coincides with a soft $\mathrm{X}$-ray background bright spot observed in the Southern hemisphere in B (130-188 eV) and C bands. According to Bochkarev (1987ab, 1990), the spot corresponds to the area of interaction of the outer part of the Local Cloud with the envelope surrounding the Sco-Cen association, namely to the position where the line of sight meets the relatively dense coronal gas near the shock front boundaries of the Sco-Cen superbubble. A similar northern maximum of $m$ probably exists (number 4 ), but there is insufficient data for its direction in the Culgoora survey.

The maximum 5 is uncertain because its position and shape vary with data combinations. It might be identified with the densest northern part of the Ori-Eri superbubble, namely with the Orion star formation region.

The minimum 7 corresponds to a very old bubble, spread by differential rotation of the Galaxy within the Galactic quadrant III. 


\section{Discussion of Time Scale of Interstellar Scintillations}

In accordance with the interpretation discussed in Section 3, the distance $L$ to the maxima 1,2 (Loop I) is about $150 \mathrm{pc}$ (Cox and Reynolds 1987; Bochkarev $1987 \mathrm{~b}, 1990$ ). The velocity of the shock wave is $530-580 \mathrm{~km} / \mathrm{s}$ (Bochkarev 1990). Adopting typical angular sizes of the point components of extragalactic radio sources at $100 \mathrm{MHz} \theta=0.2-0.5$ arcsec (see Janardhan and Alurkar 1993 and references in Slee and Siegman 1988), we find, in accordance with Rickett (1986), $\tau=L \theta / v=0.1-0.7$ years, and the size of the scattering inhomogeneities $a=20-70$ A.U.

For the maxima 3,4 , we assume the distance $L=20-40 \mathrm{pc}$ (Bochkarev $1987 \mathrm{ab}, 1990)$ and $v$ equal to the thermal velocity in coronal gas with the temperature $T=10^{6} \mathrm{~K}: v=130 \mathrm{~km} / \mathrm{s}$. In this case, $\tau=0.1-0.3$ year.

In both cases (for all most definite $m$ maxima), the estimated time scale of refractive interstellar scintillations is considerably shorter than that of the average scattering screen in the Galaxy (Rickett 1986; Slee and Siegman 1988) and is in good agreement with the observed radio-source variations.

\section{Conclusions}

A) Areas of the sky are found with intensified extragalactic radio-source interstellar scintillation at low radio frequencies $(80-160 \mathrm{MHz})$, at time scales from months to years.

B) A correspondence between the areas mentioned in Conclusion A and soft X-ray background details of large angular size is found: two Loop I bright $\mathrm{X}$-ray filaments; high Galactic latitude brightest spots in soft (B and $\mathrm{C}$ bands) $\mathrm{X}$-ray background radiation. The direction of the "interstellar absorptionfree tunnel" at the galactic longitude $l=240^{\circ}$ probably corresponds to a minimum of interstellar scintillation.

C) The estimated time scales $\tau=0.1-0.7$ year at $100 \mathrm{MHz}$ for interstellar scintillation in hot LISM structures are shorter than those for a typical scattering screen and correspond to Slee and Siegman (1988) observations.

\section{References}

Bochkarev, N.G. (1987a): Soviet Astron. 31, N 1

Bochkarev, N.G. (1987b): Astrophys. Space Sci. 138, 229

Bochkarev, N.G. (1990): Local Interstellar Medium, Moscow: Nauka Publ.

Cox, D.P., Reynolds, R.J. (1987): Ann. Rev. Astron. Astrophys. 25, 303

Janardhan, P., Alurkar, S.K. (1993): A\&A 269, 119

McCammon, D. et al. (1983): ApJ, 269, 107

Pikelner, S.B., Tsytovich, V.N. (1969): Sov. Astron. 13, N. 1

Rickett, B.J. (1986): ApJ, 307, 564

Slee, O.B., Siegman, B.C. (1988): MNRAS, 235, 1313 